\title{
Doença respiratória em menores de 5 anos no sul do Brasil: influência do ambiente doméstico'
}

\author{
Silvio O. M. Prietsch, ${ }^{2}$ Gilberto B. Fischer, ${ }^{3}$ Juraci A. César, ${ }^{2}$ \\ Berenice S. Lempek, ${ }^{2}$ Luciano V. Barbosa Jr., ${ }^{2}$ Luciano Zogbi, ${ }^{2}$ \\ Olga C. Cardoso ${ }^{2}$ e Adriana M. Santos ${ }^{2}$
}

RESUMO Objetivo. Estabelecer a prevalência de doenças agudas do trato respiratório inferior e os fatores de risco relacionados às condições de moradia em crianças de 0 a 59 meses na Cidade do Rio Grande, Estado do Rio Grande do Sul, Brasil.

Métodos. Foi realizado um estudo transversal de base populacional com 775 crianças. Foram aplicados questionários padronizados às mães ou responsáveis pelas crianças, em seus domicílios, e coletadas informações sobre condições de habitação, nível socioeconômico e tabagismo. Também foram investigados: o estado nutricional, a duração da amamentação, o atendimento pré-natal e a utilização dos serviços de saúde. As variáveis ambientais foram analisadas individualmente e em conjunto, em um escore ambiental que englobou 10 variáveis - tipo de construção, tipo de piso, aquecimento doméstico, tipo de fogão, cão no quarto da criança, cão dentro de casa, gato no quarto da criança, gato dentro de casa, aglomeração e fumo materno - variando de 0 (melhor) a 10 (pior). A análise incluiu duas etapas: bivariada, com o cálculo da razão de prevalências de cada um dos fatores de risco, e multivariada, através de regressão logística.

Resultados. A prevalência geral de doença respiratória aguda baixa foi de $23,9 \%$. Os principais fatores de risco identificados foram: escore ambiental $\geq 3$ pontos, menos de 5 anos de escolaridade materna, renda familiar mensal menor do que US\$200,00, quatro ou mais pessoas dividindo o quarto da criança e tabagismo materno. A idade materna $\geq 30$ anos foi identificada como fator de proteção.

Conclusões. É preciso implementar programas específicos de controle para as doenças respiratórias agudas na população estudada. O escore ambiental desenvolvido pode substituir as variáveis ambientais testadas, devendo ser aplicado em outros contextos para determinar a sua validade externa.

Palavras-chave Epidemiologia, infecções do trato respiratório, pré-escolar, fatores de risco, escore ambiental.

1 Estudo financiado pela Secretaria de Saúde da Prefeitura de Rio Grande (RS), Brasil.

2 Universidade Federal do Rio Grande, Departamento Materno-Infantil. Correspondência e pedidos de separatas devem ser enviados a Silvio Omar Macedo Prietsch no seguinte endereço: Rua Dr. Nascimento 396, Centro, CEP 96200-300, Rio Grande, RS, Brasil. E-mail: prietsch@uol.com.br

3 Fundação Faculdade Federal de Ciências Médicas de Porto Alegre, Porto Alegre (RS), Brasil.
Anualmente, 13 milhões de crianças menores de 5 anos morrem no mundo, das quais mais de 4 milhões morrem por doenças do trato respiratório inferior (1). Nos países mais pobres ocorrem 95\% dessas mortes, denunciando uma situação resultante da interação entre as desigualdades socioeconômicas, culturais e ambientais $(1,2)$. Na América Latina, as doenças respiratórias matam mais de 80 mil crianças por ano, e quase metade desses óbitos ocorrem no Brasil (3). No Rio Grande do Sul, as 
doenças respiratórias foram responsáveis por $20 \%$ dos cinco mil óbitos em menores de 5 anos ocorridos no ano 2000 (4).

Os principais fatores de risco ambientais para o desenvolvimento de doenças do trato respiratório inferior são a exposição a agentes poluidores domésticos, entre eles o tabagismo passivo, a agentes poluidores atmosféricos, à aglomeração e às variações climáticas (5). Nas comunidades mais pobres é freqüente o uso de combustíveis orgânicos para cozinhar ou aquecer o ambiente, muitas vezes sem o necessário cuidado com a eliminação da fumaça e de outros resíduos provenientes da combustão $(6,7)$. Essa situação é responsável por altas concentrações de poluentes nesses ambientes. A presença de alérgenos inalados, especialmente $\mathrm{o}$ ácaro do pó doméstico, componentes da saliva e pêlos de animais também estão relacionados ao incremento de resposta inflamatória da mucosa respiratória (8). Entretanto, dentre todos os poluentes domiciliares, o fumo do tabaco é o principal agente (9). Nas áreas urbanas dos países mais pobres, cerca de $30 \%$ das mulheres e quase a metade dos homens são fumantes (5). Deste fato resultam, para as crianças, altas taxas de exposição ao fumo passivo $(10,11)$.

Aos agentes poluidores domésticos somam-se, ainda, os poluentes atmosféricos, os quais apresentam associação com diversas doenças respiratórias (12). Por outro lado, a aglomeração - número de pessoas por peça do domicílio - é comum nas famílias mais pobres, nas quais a taxa de natalidade é comumente elevada e as condições de moradia são ruins (5). A aglomeração é um dos fatores de risco mais bem estabelecidos para doenças respiratórias agudas - existe clara associação en-tre as doenças respiratórias e a aglomeração ou as variáveis que se relacionam com ela, como a ordem de nascimento na família, o número de crianças menores de 5 anos no domicílio e a freqüência a creches $(5,13,14)$.

Quanto à influência da sazonalidade, está bem clara a associação de taxas mais altas de morbidade e mortalidade por doença respiratória com os meses mais frios do ano (15, 16). Não está claro, no entanto, que isso seja diretamente provocado pelo frio, sendo possível que diversos fatores ligados ao clima frio, como a aglomeração, a baixa qualidade de construção das residências e o aumento da exposição a poluentes domésticos sejam os verdadeiros responsáveis pelo incremento das doenças respiratórias no inverno (17).

Quase todos os estudos sobre fatores ambientais estão limitados por uma série de problemas metodológicos importantes, como tamanho reduzido da amostra, dificuldade na mensuração de níveis de poluentes e no estabelecimento de pontos de corte para a determinação do nível que causaria doença e influência de diversos fatores de confusão (5). Dados de morbidade respiratória devido a fatores ambientais são, ainda, afetados pela falta de padronização diagnóstica, de notificação das doenças e também pela inexistência de comunidadescontrole $(18,19)$. No Brasil, além dessas limitações, existem poucos estudos capazes de demonstrar a magnitude do problema, o que se reflete na dificuldade de planejamento e de promoção de adequadas intervenções preventivas (20).

Este estudo teve por objetivo medir a prevalência de doença aguda do trato respiratório inferior e identificar fatores de risco ambientais associados às condições de moradia na Cidade de Rio Grande, Estado do Rio Grande do Sul, Brasil. Nesse contexto, pretendeuse ainda criar um escore a fim de sintetizar o efeito dessas variáveis.

\section{MATERIAIS E MÉTODOS}

Rio Grande possui cerca de $200 \mathrm{mil}$ habitantes. A temperatura local varia de $0{ }^{\circ} \mathrm{C}$ no inverno a $36{ }^{\circ} \mathrm{C}$ no verão. A economia da cidade é baseada nas atividades portuária, pesqueira e petroquímica. Cerca de 14 mil crianças de 0 a 59 meses de idade vivem na zona urbana de Rio Grande (21).
O estudo foi realizado entre os meses de agosto e novembro de 1997. Calculou-se que, para obter uma precisão de 5\%, nível de confiança de $95 \%$ e poder de $80 \%$, seriam necessárias informações de 771 crianças (22), já incluindo um acréscimo de 10\% para compensar possíveis perdas e de $15 \%$ para controle de fatores de confusão (23).

A amostragem foi sistemática por conglomerados, feita com base nos setores censitários da Fundação Instituto Brasileiro de Geografia e Estatística (IBGE). A cada setor censitário corresponde um conjunto de 200 a 300 domicílios previamente demarcados pelo IBGE. Os dados do IBGE permitiram estimar que haveria uma criança de até 5 anos a cada quatro domicílios. Assim, para atingir o tamanho amostral desejado, seria necessário visitar 47 domicílios por setor.

Decidimos a priori que o estudo cobriria um terço dos setores censitários da zona urbana, totalizando 66 setores $(198 / 3)$. O primeiro setor foi escolhido aleatoriamente, e os demais, acrescentando-se três ao anterior. Em seguida, cada quarteirão foi numerado e foram sorteados os quarteirões para visitação. Depois disso, cada uma das esquinas dos quarteirões escolhidos foram numeradas; uma delas foi sorteada e, a partir desse ponto, sempre no sentido horário, foram visitados 47 domicílios consecutivos. Se não houvesse o número de domicílios desejados, passava-se à quadra seguinte até atingir o número necessário.

Quatorze acadêmicos voluntários dos cursos de medicina e de enfermagem e obstetrícia da Universidade Federal do Rio Grande foram treinados para aplicar questionários padronizados às mães ou responsáveis pelas crianças, pesar e medir estatura ou comprimento de menores de 5 anos. Foram utilizados dois tipos de questionários, um sobre as características das mães e famílias e outro sobre a criança. Esses questionários buscavam informações sobre características demográficas e 
reprodutivas maternas, saneamento e moradia, nível socioeconômico, utilização dos serviços de saúde, antecedentes pessoais para doenças respiratórias e sibilância, mediana de duração da amamentação, comprimento ou estatura, peso atual e ao nascer e antecedentes familiares de doença respiratória. Foi solicitada adesão do responsável ao projeto de pesquisa através de consentimento informado.

A doença respiratória aguda baixa (DRAB) foi definida pela ocorrência de tosse e dificuldade respiratória referida (respiração acelerada ou respiração difícil), com ou sem retração subcostal ou intercostal, que tivesse necessitado de tratamento médico nos últimos 7 dias. Considerou-se também, para crianças menores de 12 meses, a presença de sibilância não recorrente nos últimos 7 dias (24-27).

Foi construído um escore para avaliar a intensidade de associação das condições de moradia com o desfecho em estudo a fim de representar as circunstâncias do ambiente doméstico. O primeiro passo foi a dicotomização das variáveis representantes das condições de moradia nas categorias "adequado" e "inadequado". Considerou-se como adequada toda a situação que não oferecesse risco de exposição. No caso do tipo de construção, por exemplo, foram consideradas adequadas casas construídas em alvenaria (cimento e tijolos) ou em madeira, e inadequadas as casas com paredes de taipa, lata ou papelão. A seguir, as variáveis classificadas como "adequadas" ou "inadequadas" foram analisadas em relação ao desfecho (DRAB). Todas as variáveis incluídas no escore satisfaziam pelo menos duas das seguintes condições:

- plausibilidade biológica;

- estar inseridas na cadeia causal de infecção respiratória aguda baixa;

- ter consistência científica ampla, ou seja, ser consideradas como potencial risco para doença respiratória;

- apresentar associação com o desfecho, através de cruzamento por meio de tabela de contingência, utilizando o qui-quadrado $\left(\chi^{2}\right)$ com valor de $P$ inferior ou igual a 0,05 .

Para a categoria "adequado" foi estabelecido o valor 0 (correspondendo a 0 ponto), e para a categoria "inadequado" foi estabelecido o valor 1 (correspondendo a 1 ponto). Às condições de habitação foram adicionadas as variáveis aglomeração (número de pessoas por peça ou dormitório) e tabagismo materno, também dicotomizadas como adequadas ou inadequadas. $\mathrm{O}$ abastecimento de água e as instalações sanitárias não foram incluídos no escore.

No total, 10 variáveis compuseram o escore ambiental (tipo de construção, tipo de piso, aquecimento doméstico, tipo de fogão, cão no quarto da criança, cão dentro de casa, gato no quarto da criança, gato dentro de casa, aglomeração, fumo materno), que variou de 0 (risco mínimo) a 10 (risco máximo). A nova variável "escore ambiental" foi utilizada em um modelo de análise paralelo, substituindo as 10 variáveis originais.

Utilizou-se o programa Epi Info 6.02 (22) para a entrada dos dados, comparação de digitações e análise de consistência. No programa Statistical Package for the Social Sciences (SPSS), versão 8.0, foram feitas as análises bivariada e multivariada (28).

As cinco etapas incluídas no plano de análise são descritas a seguir. Em primeiro lugar, foi obtida uma listagem de freqüências de todas as variáveis (desfecho, fatores de risco e fatores de confusão) e exame das suas distribuições. Quando possível, foram verificadas as medidas de tendência central (média, mediana e moda) e de dispersão (amplitude e desviopadrão). A seguir, sempre que necessário, foi feita uma recodificação com definição de pontos de corte para fatores de risco e confusão.

O terceiro passo foi o cruzamento de variáveis independentes com o desfecho (DRAB). A magnitude das associações foi estimada através da razão de prevalências (prevalência nos expostos dividida pela prevalência nos não expostos) e seu intervalo de confiança de 95\% (IC95\%). Para calcular a significância estatística das associações foi utilizado o teste do $\bigotimes^{2}$ de Pearson com correção de Yates para variáveis com apenas duas categorias. Para variáveis com mais de duas categorias e ordenação lógica, utilizouse o teste para tendência linear em proporções. Depois disso, foram testadas possíveis associações entre as variáveis ambientais e o desfecho, mediante estratificação por grupos etários e renda familiar. Finalmente, realizou-se análise multivariada, controlando-se simultaneamente diversos fatores de risco ou confusão, através do método de regressão logística não condicional para obtenção das razões de prevalência e aplicação do teste de razão de verossimilhança (TRV) para obtenção da significância estatística (29).

Essa análise levou em consideração um modelo hierarquizado em que algumas variáveis são sobredeterminantes em relação às demais, conforme proposto por Victora et al. (30). Nesse modelo, pressupõe-se que o desfecho varie conforme os fatores de risco. Por exemplo, variáveis demográficas como sexo, cor e idade, embora atuem sobre o desfecho, não são influenciadas pelos outros fatores, mas podem ser determinantes sobre os demais. O nível socioeconômico exerce influência sobre as características reprodutivas maternas e sobre os fatores ambientais; por essa razão, está no primeiro nível do modelo de análise, juntamente com as variáveis demográficas. No segundo nível estão as características maternas e os fatores ambientais, que podem influenciar o processo de gravidez e as condições do parto. Nos níveis subseqüentes estão os cuidados assistenciais à gravidez e ao parto, monitoração do crescimento, variáveis nutricionais (peso ao nascer, aleitamento materno e estado nutricional), antecedentes familiares de doença respiratória e morbidade prévia. Os fatores de risco, atuando juntos ou isoladamente, têm um importante papel no estabelecimento (ou não) de DRAB. Foram analisados 
TABELA 1. Ocorrência de doença respiratória aguda baixa e razões de prevalência conforme variáveis utilizadas para a elaboração de escore ambiental. Rio Grande (RS), Brasil, 1997

\begin{tabular}{|c|c|c|c|c|}
\hline Variável & $\begin{array}{c}\text { Doença } \\
\text { respiratória } \\
\text { aguda baixa (\%) }\end{array}$ & $\begin{array}{c}\text { Razão de } \\
\text { prevalências }^{\mathrm{a}}\end{array}$ & IC95\% & $P$ \\
\hline Tipo de construção & & & & 0,005 \\
\hline Adequado & 20,2 & 1,00 & & \\
\hline Inadequado & 29,4 & 1,45 & $(1,13-1,86)$ & \\
\hline Tipo de piso & & & & 0,05 \\
\hline Adequado & 21,9 & 1,00 & & \\
\hline Inadequado & 28,0 & 1,28 & $(0,99-1,65)$ & \\
\hline Aquecimento doméstico & & & & 0,11 \\
\hline Adequado & 18,5 & 1,00 & & \\
\hline Inadequado & 25,2 & 1,36 & $(0,95-1,95)$ & \\
\hline Tipo de fogão & & & & 0,34 \\
\hline Adequado & 23,3 & 1,00 & & \\
\hline Inadequado & 29,2 & 1,25 & $(0,85-1,84)$ & \\
\hline Cão no quarto da criança & & & & 0,20 \\
\hline Não (adequado) & 23,7 & 1,00 & & \\
\hline Sim (inadequado) & 40,0 & 1,69 & $(0,78-3,65)$ & \\
\hline Cão dentro de casa & & & & 0,03 \\
\hline Não (adequado) & 22,8 & 1,00 & & \\
\hline Sim (inadequado) & 35,4 & 1,55 & $(1,09-2,21)$ & \\
\hline Gato no quarto da criança & & & & 0,33 \\
\hline Não (adequado) & 23,7 & 1,00 & & \\
\hline Sim (inadequado) & 31,3 & 1,32 & $(0,63-2,76)$ & \\
\hline Gato dentro de casa & & & & 0,85 \\
\hline Não (adequado) & 23,7 & 1,00 & & \\
\hline Sim (inadequado) & 25,0 & 1,06 & $(0,74-1,50)$ & \\
\hline Aglomeração (pessoa/quarto) & & & & 0,001 \\
\hline Adequado (até 2 pessoas) & 17,5 & 1,00 & & \\
\hline Inadequado ( 3 ou mais pessoas) & 27,5 & 1,63 & $(1,13-2,09)$ & \\
\hline Fumo materno & & & & 0,001 \\
\hline Não fuma (adequado) & 20,8 & 1,00 & & \\
\hline Fuma (inadequado) & 28,6 & 1,38 & $(1,05-1,97)$ & \\
\hline Total $(n=775)$ & 23,9 & & & \\
\hline
\end{tabular}

${ }^{a}$ Prevalência nos expostos dividida pela prevalência nos não expostos.

todos os fatores de risco com valor de $P \leq 0,20$.

\section{RESULTADOS}

Setecentas e noventa e duas crianças menores de 5 anos foram identificadas em 3185 domicílios visitados. Dessas, 775 foram incluídas no estudo, 13 não foram encontradas e quatro se recusaram a participar. Esses dados contabilizaram perdas de $2,1 \%$.

A distribuição das crianças foi semelhante quanto à faixa etária e ao sexo, e $83 \%$ tinham cor branca. Metade das famílias possuía cinco ou mais membros; em um terço havia pelo menos dois menores de 5 anos. Sete por cento das mães e $4 \%$ dos pais não sabiam ler ou escrever. A renda mensal de uma em cada seis famílias era igual ou inferior a US\$ 100,00. Cerca de 30\% tinham renda mensal menor do que US\$200,00.

Setenta e dois por cento das famílias moravam em casas de alvenaria (tijolos), 27,4\% em casas de madeira e 9,6\% em casas construídas com lata, plástico ou papelão. Noventa e oito por cento dos domicílios recebiam água tratada e 90\% eram atendidos por rede de esgoto. Fogão a gás era utilizado por $90 \%$ das famílias, enquanto que $10 \%$ utilizavam fogão a lenha; $75 \%$ das residências não dispunham de qualquer equipamento para o aquecimento doméstico.

Em metade dos domicílios havia duas ou mais pessoas por peça, e $66 \%$ deles abrigavam três ou mais pessoas por dormitório. A maioria das famílias tinha animais domésticos, com a predominância de cães, presentes em $62 \%$ dos domicílios. Em $63 \%$ dos domicílios havia pelo menos um fumante. Entre os pais, $40 \%$ eram fumantes, assim como $37 \%$ das mães. As mães fumavam em média 14 cigarros por dia [desvio-padrão (DP) = $8,1]$, e os pais fumavam em média 17 cigarros (DP $=9,4)$.

A prevalência geral de DRAB foi de 23,9\% (IC95\%: 21,1-26,9). A tabela 1 mostra a ocorrência de DRAB conforme as variáveis relativas às condições de habitação, que foram utilizadas para a elaboração do escore ambiental. A distribuição das famílias quanto à pontuação do escore foi: $0=$ $4,9 \% ; 1=13,7 \% ; 2=21,3 \% ; 3=20 \%$; $4=16,7 \% ; 5=12,8 \% ; 6=3,3 \% ; 7=$ $3,0 \% ; 8=2,1 \% ; 9=1,9 \%$; e $10=0,3 \%$.

$\mathrm{Na}$ análise bivariada, estiveram significativamente associadas com a ocorrência de DRAB as seguintes variáveis: escolaridade materna, escolaridade paterna, renda familiar, idade materna ao nascimento da criança, fumo durante a gravidez, aglomeração (pessoas/peça e pessoas/dormitório), fumo materno, número de fumantes no domicílio e mais de 3 pontos no escore ambiental.

A tabela 2 mostra as variáveis introduzidas no primeiro nível do modelo de regressão logística. $\mathrm{O}$ risco de DRAB foi $65 \%$ maior em crianças que tinham mães com menor nível de escolaridade e 50\% maior em famílias com renda mensal inferior a US\$ 200,00 . Na tabela 3 observamos as variáveis introduzidas no segundo nível. Parir com 30 anos ou mais apareceu como um fator protetor à ocorrência de DRAB nos filhos, enquanto aglomeração familiar e fumo materno apresentaram um claro efeito dose-resposta: quanto maior o número de pessoas no domicílio e a quantidade de cigarros fumados pela mãe, maior o risco de DRAB. 
TABELA 2. Análise multivariada por regressão logística da associação entre doença respiratória aguda baixa, cor da criança e variáveis socioeconômicas. Rio Grande (RS), Brasil, 1997

\begin{tabular}{lcc}
\hline & \multicolumn{2}{c}{ Razão de prevalências (IC95\%) } \\
\cline { 2 - 3 } Variáveis (modelo hierárquico) & Bruta & Ajustada $^{\mathrm{b}}$ \\
\hline Cor da criança & & \\
$\quad$ Branca & 1,00 & 1,00 \\
$\quad$ Parda e preta & $1,29(0,96-1,74)$ & $1,18(0,86-1,61)$ \\
TRV c categórico & $P=0,13$ & $P=0,30$ \\
Escolaridade materna (anos completos) & $1,70(1,18-2,44)$ & $1,65(1,15-2,37)$ \\
0 a 4 & $1,49(1,04-2,12)$ & $1,47(1,04-2,08)$ \\
5 a 8 & 1,00 & 1,00 \\
9 e mais & $P=0,01$ & $P=0,02$ \\
TRV categórico & $P=0,004$ & $P=0,01$ \\
TRV tendência linear & & \\
Escolaridade paterna (anos completos) & $1,54(1,04-2,27)$ & $1,33(0,92-1,92)$ \\
0 a 4 & $1,45(1,00-2,10)$ & $1,32(0,90-1,93)$ \\
5 a 8 & 1,00 & 1,00 \\
9 e mais & $P=0,06$ & $P=0,35$ \\
TRV categórico & $P=0,03$ & $P=0,22$ \\
TRV tendência linear & & $1,50(1,02-2,20)$ \\
Renda familiar (dólares americanos) & $1,73(1,25-2,40)$ & $1,23(0,96-1,57)$ \\
Menos de 200 & $1,37(0,99-1,88)$ & 1,00 \\
200 a 500 & 1,00 & $P=0,12$ \\
Mais de 500 & $P=0,004$ & $P=0,04$ \\
TRV categórico & $P=0,001$ & \\
TRV tendência linear & & \\
\hline
\end{tabular}

a Prevalência nos expostos dividida pela prevalência nos não expostos.

${ }^{b}$ Ajustada por cor da pele, escolaridade materna, escolaridade paterna e renda familiar.

${ }^{c} \mathrm{TRV}=$ Teste de razão de verossimilhança

TABELA 3. Análise multivariada por regressão logística da associação entre doença respiratória aguda baixa, idade materna e variáveis ambientais. Rio Grande (RS), Brasil, 1997

\begin{tabular}{lcc}
\hline & \multicolumn{2}{c}{ Razão de prevalências $^{\mathrm{a}}$ (IC95\%) } \\
\cline { 2 - 3 } Variáveis (modelo hierárquico) & Bruta & Ajustada $^{\mathrm{b}}$ \\
\hline Idade materna ao nascimento (anos) & & \\
13 a 19 & $1,06(0,78-1,43)$ & $1,01(0,87-1,17)$ \\
20 a 29 & 1,00 & 1,00 \\
30 e mais & $0,73(0,53-1,00)$ & $0,70(0,50-0,98)$ \\
TRV categórico & $P=0,08$ & $P=0,07$ \\
TRV tendência linear & $P=0,04$ & $P=0,05$ \\
Aglomeração (pessoas/peça) & & 1,00 \\
1 ou menos & 1,00 & $1,17(0,77-1,77)$ \\
2 & $1,32(1,00-1,74)$ & $1,21(0,91-1,60)$ \\
3 ou mais & $1,36(0,94-1,96)$ & $P=0,41$ \\
TRV categórico & $P=0,09$ & $P=0,35$ \\
TRV tendência linear & $P=0,05$ & 1,00 \\
Aglomeração (pessoa/dormitório) & 1,00 & $1,33(0,96-1,85)$ \\
2 ou menos & $1,43(1,03-1,99)$ & $1,54(1,08-2,19)$ \\
3 & $1,72(1,23-2,41)$ & $P=0,05$ \\
4 ou mais & $P=0,005$ & $P=0,02$ \\
TRV categórico & $P=0,001$ & 1,00 \\
TRV tendência linear & & $1,22(0,80-1,86)$ \\
Fumo materno (cigarros/dia) & 1,00 & $P=0,05$ \\
Não fuma & $1,33(0,89-1,99)$ & $P=0,01$ \\
1 a 9 & $1,43(1,09-1,87)$ & $P=0,03$ \\
10 ou mais & $P=0,001$ & $1,41(1,07-1,86)$ \\
TRV categórico & & \\
TRV tendência linear & & \\
\hline
\end{tabular}

a Prevalência nos expostos dividida pela prevalência nos não expostos.

${ }^{\mathrm{b}}$ Ajustada por escolaridade materna, renda familiar, idade materna, pessoas/peça, pessoas/quarto de dormir e fumo materno. ${ }^{\mathrm{c}} \mathrm{TRV}=$ Teste de razão de verossimilhança.
Ao estudar a associação do escore ambiental com doença respiratória, estratificada por grupos de renda familiar, não foram observadas diferenças (figura 1). A figura 2 mostra os resultados da análise da variável "escore ambiental" bruta e ajustada para os fatores socioeconômicos (nível superior) e idade materna (mesmo nível). Foi observada uma associação direta entre o número de pontos obtidos no escore e doença respiratória: quanto maior o número de pontos obtidos no escore, maior o risco de DRAB. Para aquelas crianças com escore ambiental igual ou superior a 5 , o risco de ocorrência de DRAB foi cerca de duas vezes maior, mesmo após o ajuste para todas as variáveis estudadas.

\section{DISCUSSÃO}

A prevalência de doença respiratória aguda do trato inferior foi bastante elevada neste estudo. O baixo nível de escolaridade materna, a aglomeração familiar, o tabagismo e as precárias condições de moradia foram importantes determinantes da ocorrência de doença.

Ao discutir esses resultados, é preciso ter em mente que $o$ delineamento transversal não é o mais apropriado para medir relação de causalidade. No entanto, foi utilizado pelo baixo custo, rapidez, facilidade de execução e, especialmente, por necessidade de subsidiar um programa específico de atenção às doenças respiratórias nos primeiros anos de vida (31). É possível, também, que o período do ano no qual os dados foram coletados (final do inverno e início da primavera) tenha influenciado a prevalência observada. Além disso, o critério diagnóstico de DRAB, aplicado neste estudo, apresenta alta sensibilidade e baixa especificidade, o que pode superestimar a ocorrência de doença (24). Todavia, é pouco provável que esta limitação tenha afetado tanto a prevalência de doença respiratória quanto os fatores de risco a ela associados. 
FIGURA 1. Escore ambiental e prevalência de doença respiratória aguda baixa conforme renda familiar. Rio Grande (RS), Brasil, $1997^{\mathrm{a}}$

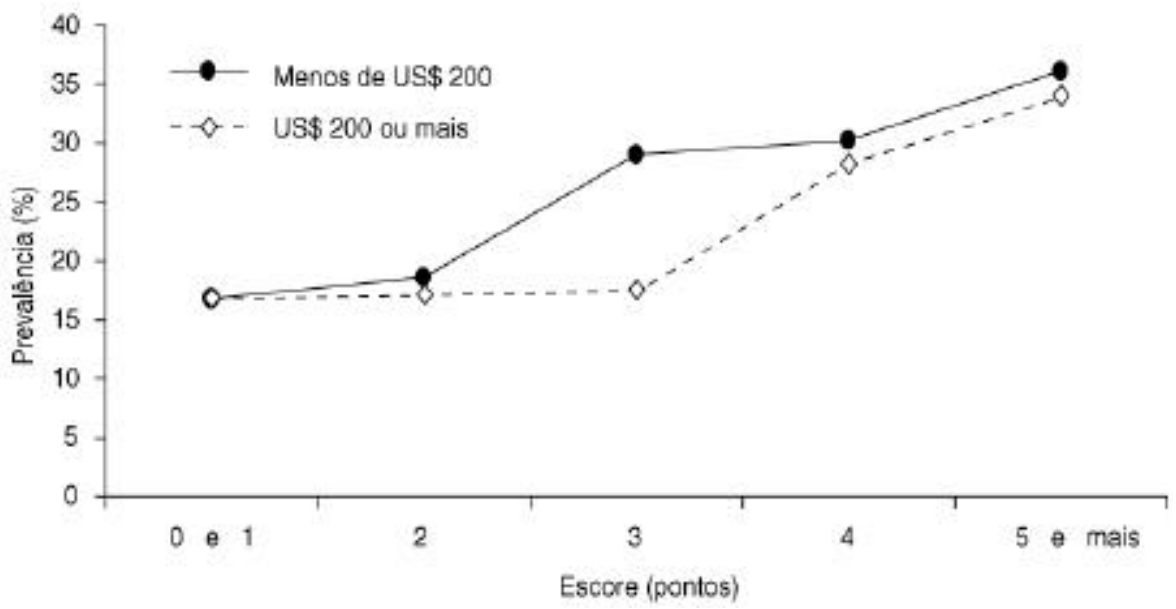

${ }^{a}$ Melhor escore ambiental $=0$; pior $=10$. As variáveis tipo de construção, tipo de piso, aquecimento doméstico, tipo de fogão, cão no quarto da criança, cão dentro de casa, gato no quarto da criança, gato dentro de casa, aglomeração e fumo materno foram utilizadas para elaboração do escore.

FIGURA 2. Risco de doença respiratória aguda baixa conforme pontuação no escore ambiental. Rio Grande (RS), Brasil, 1997a

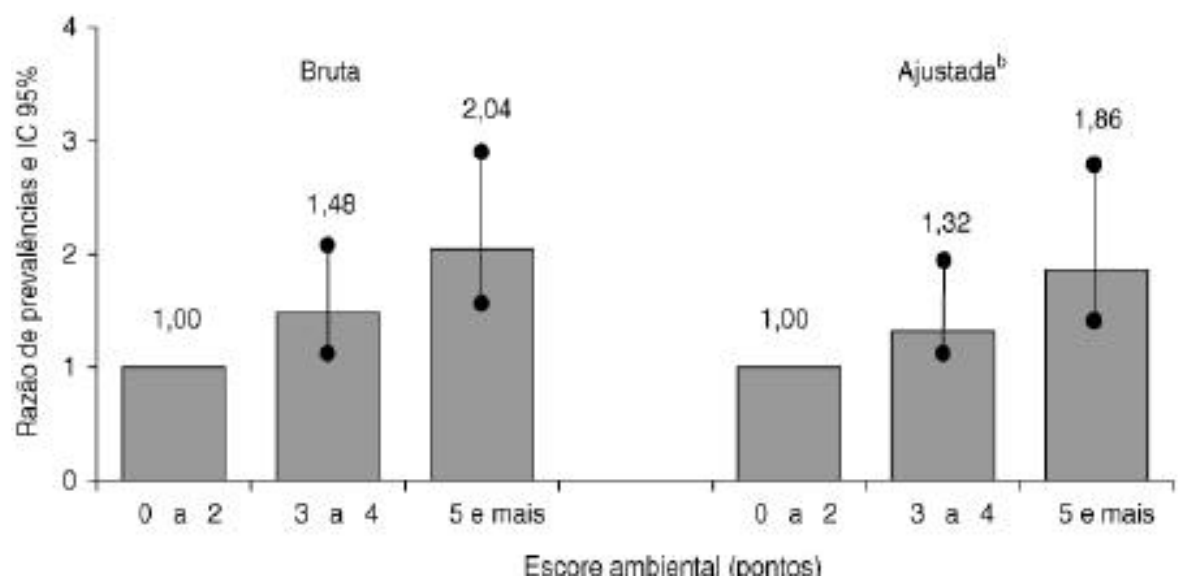

a Melhor escore ambiental $=0$; pior $=10$. As variáveis tipo de construção, tipo de piso, aquecimento doméstico, tipo de fogão, cão no quarto da criança, cão dentro de casa, gato no quarto da criança, gato dentro de casa, aglomeração e fumo materno foram utilizadas para elaboração do escore.

${ }^{\mathrm{b}}$ Ajustada por escolaridade de mãe, renda familiar e idade materna.

Quanto às variáveis socioeconômicas, a escolaridade materna manteve a significância estatística após o ajuste para as variáveis demográficas e outras variáveis socioeconômicas, reforçando a idéia de que este é um importante determinante das condições de saúde das crianças (32). Como em outros estudos, encontramos um efeito protetor para os filhos de na ajustada pelos fatores socioeconômicos, idade materna e demais variáveis ambientais. Tal resultado indica que a aglomeração, embora tenha muito de seu efeito confundido por fatores socioeconômicos e ambientais, desem-penha um papel independente como fator de risco para doenças respiratórias agudas, especialmente quanto ao número de pessoas que compartilham o quarto de dormir com as crianças. Quanto ao tabagismo, encontramos uma alta prevalência do hábito de fumar nessa população, especialmente das mães, e uma importante associação de fumo materno com DRAB. $\mathrm{Na}$ análise multivariada, após o controle para idade materna, outras variáveis ambientais e nível socioeconômico, o tabagismo materno permaneceu estatisticamente significativo. Essas associações encontram-se descritas em outra publicação e corroboram a influência de tais fatores sobre a saúde respiratória das crianças (32).

A grande variabilidade dos fatores envolvidos na determinação das condições de habitação causa uma série de dificuldades de análise. Ao tipo de construção da casa e condições de saneamento básico somam-se fatores tão diversos como a presença de animais domésticos, uso de equipamentos para aquecimento, hábitos de manutenção e limpeza, entre outros. Muitos desses fatores estão fortemente ligados às condições socioeconômicas, como o saneamento, mas outros, como a presença de animais dentro de casa, não são - a princípio - dependentes de nenhuma outra condição. Estudar essas variáveis uma a uma é sempre uma tarefa difícil e, com freqüência, os resultados obtidos são confundidos ou confundem outros fatores. Os estudos sobre doenças respiratórias agudas têm analisado esses fatores de diversas formas. López Bravo et al. mostraram uma associação significativa entre o número de episódios de doenças respiratórias agudas com condições domésticas desfavoráveis (35). Victora et al., estudando os fatores de risco para pneumonia, desenvolveram um escore para classificar a qualidade da 
moradia, mas não houve associação significativa deste com o desfecho (36).

$\mathrm{O}$ escore ambiental criado neste estudo permitiu estudar, em conjunto, os fatores do ambiente doméstico. As variáveis representativas de aglomeração e tabagismo certamente foram as que determinaram maior efeito entre as constituintes do escore, mas não poderiam deixar de ser acrescentadas às condições de moradia, pois são importantes componentes do ambiente doméstico. As variáveis abastecimento de água e instalações sanitárias não foram incluídas no escore porque quase a totalidade das famílias dispunham de ambas, de forma adequada. Na análise multivariada não foi possível testar simultaneamente as variáveis de condição de moradia, aglomeração, fumo materno e escore ambiental. Isso porque o modelo de regressão logística utilizado não permite a análise

1. World Health Organization (WHO). Technical bases for WHO recommendations on the management of pneumonia in children at first-level health facilities. Interim Programme Report 1991. Geneva: WHO; 1993. (WHO/ARI/91.20).

2. Benguigui Y. Controle das infecções respiratórias agudas: implementação, acompanhamento e avaliação. Vol 2 . Washington, D.C.: Organização PanAmericana da Saúde; 1997. Pp. 1-15.

3. Arias SJ, Benguigui Y, Bossio JC. Infecciones respiratorias agudas en las Américas: magnitud, tendencia y avances en el control. Washington, D.C.: Organización Panamericana de la Salud; 1992.

4. Secretaria de Saúde e do Meio Ambiente do Rio Grande do Sul (SSMARGS). Vol 25. Estatísticas de saúde: mortalidade 2000. Porto Alegre: SSMARGS; 2001.

5. Victora CG. Factores de riesgo en las IRA bajas. En: Benguigui Y, López-Antuñano FJ, Yunes J. Infecciones respiratorias en niños. Washington, D.C.: Organización Panamericana de la Salud; 1996. Pp. 45-63.

6. Pandey MR, Smith KR, Boleij JSM, Wafula EM. Indoor air pollution in developing countries and acute respiratory infections in children. Lancet 1989;1(8635):427-429.

7. Johnson AW, Aderele WI. The association of household pollutants and socio-economic risk factors with the short-term outcome of acute lower respiratory infections in hospitalized pre-school Nigerian children. Ann Trop Paediatr 1992;12(4):421-432.

8. Phelan PD, Olinsky A, Robertson CF. Respiratory illness in children. 4th ed. simultânea de variáveis idênticas, uma vez que a variável do escore ambiental engloba condições de moradia, aglomeração e fumo materno. Por esse motivo, utilizamos um modelo no qual o escore ambiental foi introduzido no segundo nível, juntamente com a idade materna. Comparando os dois modelos construídos para a análise, o primeiro com os fatores ambientais e o segundo com a variável escore ambiental substituindo esses fatores, observamos que não houve diferenças entre eles, inclusive quanto à análise dos níveis proximais ao desfecho.

Os dados deste estudo mostram a necessidade de melhorar a distribuição de renda e garantir amplo acesso à escola, em especial às mães. Com isso, a prevalência de doenças respiratórias nas crianças seria reduzida, principalmente através de melhores condições de moradia,

\section{REFERÊNCIAS}

Australia: Blackwell Scientific Publication; 1994. Pp. 27-51.

9. American Academy of Pediatrics. Environmental tobacco smoke: a hazard to children - Committee on Environmental Health. Pediatrics 1997;99(4):639-642.

10. Lister SM, Jorm LR. Parental smoking and respiratory illnesses in Australian children aged 0-4 years: ABS 1998-90 National Health Survey results. Aust N Z J Public Health 1998; 22(7):781-786.

11. Gergen PJ, Fowler JA, Maurer KR, Davis WW, Overpeck MD. The burden of environmental tobacco smoke exposure on the respiratory health of children 2 months through 5 years of age in the United States: Third National Health and Nutrition Examination Survey, 1988 to 1994. Pediatrics 1998;101(2):E8.

12. Bascom R, Bromberg PA, Hill C, Costa DA, Devlin R, Dockery DW, et al. Health effects of outdoor air pollution. Committee of the Environmental and Occupational Health Assembly of the American Thoracic Society. Am J Respir Crit Care Med 1996;153(1):3-50.

13. Schmunis G. Prólogo. Em: Benguigui Y. Investigações operacionais sobre o controle das infecções respiratórias agudas (IRA). Washington, D.C.: Organização PanAmericana da Saúde; 1997. Pp. iii-iv.

14. Victora CG, Barros FC, Vaughan JP. Epidemiologia da desigualdade: um estudo longitudinal de 6000 crianças brasileiras. 2a ed. São Paulo: Hucitec; 1988.

15. Victora CG, Vaughan JP, Barros FC. The seasonality of infant deaths due to diarrheal and respiratory disease in southern Brazil, menor aglomeração familiar, e redução do hábito de fumar.

Quanto ao escore ambiental desenvolvido, é possível afirmar que este escore pode substituir todas as variáveis ambientais testadas neste trabalho, podendo ser utilizado em outros estudos seqüenciais nesta população, necessários para a implementação de programas específicos de controle das doenças respiratórias agudas. Seria interessante também a aplicação do escore em outras situações de pesquisa e em outros contextos, com os ajustes pertinentes, para determinar a sua validade externa, especialmente considerando o manejo simples desse instrumento em relação à qualidade da informação que propicia.

1974- 1978. Bull Pan Am Health Organ 1985;19(1): 29-39.

16. Zaman K, Baqui AH, Yunus M, Sack RB, Bateman OM, Chowdhury HR, et al. Acute respiratory infections in children: a community-based longitudinal study in rural Bangladesh. J Trop Pediatr 1997;43(3):133-137.

17. Hortal M, Benitez A, Contera M, Etorena P, Montano A, Meny M. A community-based study of acute respiratory tract infections in children in Uruguay. Rev Infect Dis 1990;12 (Suppl 8):966-973.

18. Deb SK. Acute respiratory disease survey in Tripura in case of children below five years of age. J Indian Med Assoc 1998;96(4):111-116.

19. Selwyn BJ. The epidemiology of acute respiratory tract infection in young children: comparison of findings from several developing countries. Coordinated Data Group of BOSTID Researchers. Rev Infect Dis 1990;12 (Suppl 8):S870-888.

20. Barros FC, Victora CG. Epidemiologia da saúde infantil: um manual para diagnósticos comunitários. $2^{\mathrm{a}}$ ed. São Paulo: Hucitec/ UNICEF; 1994.

21. Indicadores sociais: crianças \& adolescentes. Censo demográfico, Rio Grande do Sul, 1991. Brasília: UNICEF/IBGE; 1994. Pp. 19-40.

22. Dean AG, Dean JA, Coulombier D, Brendel KA, Smith DC, Burton AM. Epi Info, Version 6.02: a word processing, database and statistics program for epidemiology on microcomputers. Atlanta (GA): Centers for Disease Control and Prevention; 1994.

23. Smith PG, Day NE. The design of case-control studies: the influence of confounding and 
interaction effects. Int J Epidemiol 1984;13(3): 356-365.

24. Samet JM, Cushing AH, Lambert WE, Hunt WC, McLaren LC, Young SA, et al. Comparability of parent reports of respiratory illnesses with clinical diagnoses in infants. Am Rev Respir Dis 1993;148(2):441-446.

25. Ferris BG. Epidemiology Standardization Project (American Thoracic Society). Am Rev Respir Dis 1978;118(6 Pt 2):1-120.

26. World Health Organization (WHO). Programme for control of acute respiratory infections: definitions of clinical signs and symptoms. Interim Programme Report Draft 1988. Geneva: WHO; 1988.

27. Falade AG, Tschäppeler H, Greenwood BM, Mulholland EK. Use of simple clinical signs to predict pneumonia in young Gambian children: the influence of malnutrition. Bull World Health Organ 1995;73(3):299-304.
28. Norussis M. SPSS for Windows, Version 8.0. Chicago: SPSS Inc.; 1998.

29. Rothman KJ. Modern epidemiology. Boston: Little, Brown and Co.; 1986.

30. Victora CG, Huttly SR, Fuchs SC, Olinto MTA. The role of conceptual frameworks in epidemiological analysis: a hierarchical approach. Int J Epidemiol 1997;26(1):224-227.

31. Beaglehole R, Bonita R, Kjellström T. Epidemiologia básica. São Paulo: Editora Santos; 1996.

32. Prietsch SOM, Fischer GB, Cesar JA, Fabris AR, Mehanna H, Ferreira THP, et al. Doença aguda das vias aéreas inferiores: influência do ambiente doméstico e do tabagismo materno. J Pediatr (Rio J) 2002;78(5):415-422.

33. Fonseca WVC. Risk factors for childhood pneumonia among the poor urban in Fortaleza, Brazil: a case-control study. [Doctoral dissertation]. London: London School of Hygiene and Tropical Medicine; 1993.
34. Rahman MM, Rahman AM. Prevalence of acute respiratory tract infection and its risk factors in under five children. Bangladesh Med Res Counc Bull 1997;23(2):47-50.

35. López Bravo IM, Sepúlveda H, Valdés I. Acute respiratory illnesses in the first 18 months of life. Rev Panam Salud Publica 1997; $1(1): 9-17$.

36. Victora CG, Fuchs SC, Flores JAC, Fonseca W, Kirkwood B. Risk factors for pneumonia among children in a Brazilian metropolitan area. Pediatrics 1994;93(6):977-985.

Manuscrito recebido em 25 de março de 2002. Aceito em versão revisada em 11 de outubro de 2002.

ABSTRACT Objective. To determine the prevalence of acute lower respiratory tract infections and the risk factors associated with living conditions among children up to 5 years of age in the city of Rio Grande, in the state of Rio Grande do Sul, Brazil.

Respiratory illnesses $\begin{aligned} & \text { Methods. A population-based cross-sectional study was carried out with } 775 \text { children. A } \\ & \text { standardized questionnaire was administered to the mother or other caregiver at the child's home }\end{aligned}$ in children younger in order to collect information on housing conditions, socioeconomic status, and smoking in the than 5 years of age in home. Additional variables examined included nutritional status, duration of breast-feeding, southern Brazil: the prenatal care, and utilization of health care services. Environmental variables were analyzed influence of the home environment individually and were also grouped together in an "environmental score" that encompassed 10 variables: type of house construction, type of floor, home heating system, type of stove, dog in the child's room, dog in the house, cat in the child's room, cat in the house, number of people per room, and maternal smoking. The grouped environmental score ranged from 0 (best) to 10 (worst). The analysis included two stages: a bivariate stage, in which the prevalence ratio was calculated for each risk factor, and a multivariate stage, with logistic regression.

Results. The overall prevalence of acute lower respiratory tract infections was $23.9 \%$. The main risk factors identified were: environmental score $\geq 3$ points, maternal schooling $<5$ years, monthly family income $<$ US\$ 200, four or more people sharing the child's bedroom, and maternal smoking. Maternal age $\geq 30$ years was found to protect against the development of respiratory illness.

Conclusions. Specific programs need to be implemented to control acute respiratory illnesses in the population studied. In future studies with this population, the environmental score that we developed could be used in place of the complete set of environmental variables that we tested. This environmental score should be applied in other contexts so as to determine its external validity.

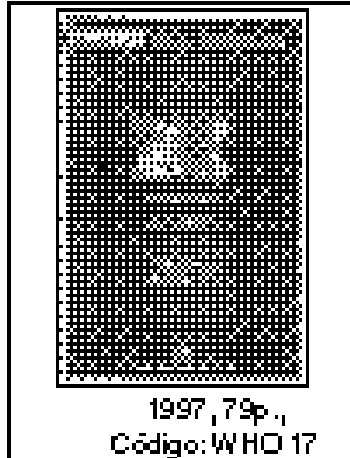

Precio: US 11001 US 800 en Améria Laina yed Caribe

\section{Tratamiento de la Tuberalosis. Directrices para los programas nacionales, 2a ed}

En esta cobra se facilitan mormas prácticas for mulacks por expertos para el trata miento y cortrol de la erfer medad en al contexto de los programas antituberculosos nacionales. Esta segunda edción del manual ha sido rerisada para reflejar la gran experiencia adguirida, desde 1993, con el uso de las estrategas de contrd recomendactas por la Organización Mundial de la Salud.

El marual drece a los directores de programas, a los encargados de formular las políticas y a los médicos en ejercicio clírico un método claro y de utilidad comprobada para el cortrol de la tuberculosis que depende de definiciones de caso precisas, cate gorías terapéuticas bien definidas y regímenes de trata miento estandarizados a base de medcamentos artituberculosos esenciales.

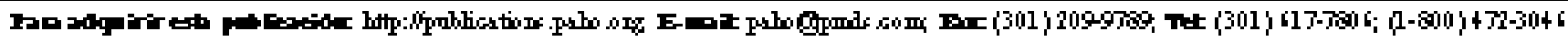

